\title{
L'ART DE NE PAS RÉINVENTER LA ROUE : MENER À BIEN UN PROJET DE RÉDACTION SCIENTIFIQUE
}

\author{
Nadine FORGET-DUBOIS ${ }^{1 *}$ \\ ${ }^{1}$ La Plume scientifique (laplumescientifique.com), Qc, Canada \\ *laplume@laplumescientifique.com
}

\section{Pour citer l'article}

Forget-Dubois, N. (2020). L'art de ne pas réinventer la roue: mener à bien un projet de rédaction scientifique. Psycause: Revue scientifique étudiante de l'École de psychologie de I'Université Laval, 10(1), 61-71.

\section{Droits d'auteur}

(C) 2020 Forget-Dubois. Cet article est distribué en libre accès selon les termes d'une licence Creative Commons Attribution 4.0 International (de type CC-BY 4.0) qui permet l'utilisation du contenu des articles publiés de façon libre, tant que chaque auteur ou autrice du document original à la publication de l'article soit cité(e) et référencé(e) de façon appropriée. 


\title{
L'ART DE NE PAS RÉINVENTER LA ROUE : MENER À BIEN UN PROJET DE RÉDACTION SCIENTIFIQUE
}

\author{
Nadine FORGET-DUBOIS ${ }^{1 *}$ \\ 'La Plume scientifique (laplumescientifique.com), Qc, Canada \\ *laplume@laplumescientifique.com
}

Écrire une thèse, un mémoire ou un article scientifique exige d'atteindre un niveau de rédaction professionnel pour lequel vos études précédentes vous ont, pour la plupart, mal préparé(e)s. Si certain(e)s apprennent par imitation, d'autres, la majorité sans doute, peinent à rédiger et en éprouvent de la détresse. Pourtant, écrire un texte scientifique de haut niveau s'apprend, comme n'importe quelle autre habileté nécessaire à la complétion d'un projet de recherche. Toutefois, vous devrez l'apprendre largement par vous-même et le but de cet article est de vous y aider. Le succès de votre écrit scientifique se joue sur deux plans: d'abord, la planification et la structuration du texte sous forme d'arc narratif, c'est-àdire sous forme d'histoire avec un début, un milieu, une fin et une intrigue. Ensuite, la gestion de vos comportements d'écriture: mettre en place les conditions favorables qui vous conviennent. Je vous propose de découvrir quelques principes de rédaction pour soutenir votre auto-apprentissage de la rédaction scientifique. Le temps investi initialement dans la maîtrise de l'écriture scientifique se traduit en économie de temps pendant la rédaction et la révision avec votre superviseur(e). Vous pourriez même finir par y prendre plaisir.

Si la rigueur reste la première caractéristique d'un écrit scientifique, la rédaction de votre thèse, mémoire ou article scientifique représente aussi un acte créatif. Une question de recherche peut toujours être contextualisée de multiples manières valables. Par exemple, si vous étudiez les conséquences de la consommation précoce de cannabis, vous pouvez tout autant décrire ses conséquences psychosociales que ses conséquences cognitives ou celles sur les pathologies pulmonaires. Cependant, vous ne pourriez pas rapporter ces trois points de vue dans le même texte: il faut décider si vous étudiez la psychologie ou la médecine. Impossible de raconter toutes les histoires que les données suggèrent, vous devez en choisir une seule. Et c'est là, dans le point de vue original que vous allez apporter à la littérature scienti- fique, que se trouve l'aspect créatif de votre écrit. La première erreur, en planifiant votre rédaction, serait de ne pas choisir quelle histoire vous allez raconter à partir de vos questions et de vos résultats. Écrire, c'est aussi décider ce qu'on ne va pas écrire. Alors comment trouver un fil conducteur dans le fouillis de vos lectures, données, analyses et résultats? Et comment arriver au bout de sa rédaction sans d'abord refaire les erreurs que tout(e)s les autres étudiant(e)s du monde ont faites avant vous?

Une petite mise en garde s'impose avant de continuer. Rédiger est un acte très personnel et aucune recette ne saurait s'appliquer en toutes circonstances. Toutefois, comme le modèle de texte scientifique classique ne permet pas beaucoup de variation sur la formule 
Introduction - Méthode - Résultats - Discussion, les techniques de structuration du texte que je vais présenter ici devraient s'appliquer à de nombreux projets de rédaction sans nécessiter beaucoup d'adaptation. Même les recherches qualitatives s'articulent selon les structures que je vous propose avec quelques aménagements. Le principe est d'apprendre des méthodes de ceux et celles qui sont passés avant vous et de vous en inspirer pour découvrir la vôtre. II faut pour cela prendre conscience de l'existence des nombreuses ressources tragiquement sousemployées qui existent sur tous les aspects de l'écriture scientifique. Lorsque j'ai autrefois attaqué la rédaction de ma thèse, dans un mélange d'anxiété, de paresse et d'esprit pratique, je me suis dit que sûrement, quelqu'un pouvait me montrer comment faire? Aucun professeur ne m'a donné mieux que des conseils généraux, il n'y avait aucun cours de rédaction. Toutefois, j'ai découvert par moi-même toute une tablette de livres exauçant mon souhait à la bibliothèque de mon université. J'ai bricolé avec leur aide une manière de gérer la rédaction convenant à ma personnalité. Dans l'ensemble, j'ai aimé rédiger ma thèse: je me sentais en contrôle de la tâche, ceci expliquant sans doute cela. Mon but ici est donc de vous donner un coup de pouce pour que vous viviez aussi une expérience positive de rédaction.

\section{Trouver l'histoire à raconter}

Selon le chercheur en psychologie Scott Barry Kaufmann, les personnes créatives sont capables de pensée divergente pour faire surgir leurs idées et de pensée convergente pour s'atteler à la longue tâche d'en choisir une et de la mener à sa réalisation (Kaufman \& Gregoire, 2015). Pour trouver le fil conducteur de votre écrit scientifique, vous avez besoin de jouer avec les idées. Jouer, comme dans avoir du plaisir. Vous êtes des étudiant(e)s aux cycles supérieurs, n'allez pas me dire que vous n'aimez pas jongler avec des idées compliquées... La méthode que je vous propose consiste à chercher la nature des liens entre les idées principales qui feront partie de votre thèse, mémoire ou article pour dégager la structure du texte. Le bon moment pour la mettre en pratique? Après avoir absorbé la littérature scientifique se rapportant à votre sujet. Pour écrire votre projet de recherche, la lecture suffit. Pour planifier l'écrit final, attendez d'avoir des résultats très avancés afin d'anticiper la discussion.

Il y a donc un temps pour la créativité et la pensée divergente. Fermez votre traitement de texte, car celui-ci vous force à une pensée linéaire. Rappelez-vous pour quelle raison votre question de recherche est importante. Oui, elle l'est. Jetez sur papier (ou tablette si l'absence de technologie vous trouble) les principales idées en lien avec les questions de recherche et le contexte théorique qui leur a donné lieu. Ces idées sont les personnages de votre histoire. Que racontent-elles? Représentez visuellement avec des flèches et des ensembles la nature de leurs liens: est-ce qu'elles se complètent? S'opposent? Vont dans toutes les directions? Dans une histoire, les liens causaux entre les événements rapportés constituent l'intrigue. Bon nombre d'écrits scientifiques peuvent se classer dans trois catégories d'intrigue, à garder en tête lorsque vous cherchez l'histoire à raconter (voir Figure 1):

1) La quête. Parfois, les découvertes scientifiques s'enchaînent dans une belle continuité. Votre écrit doit donc s'inscrire dans la suite. Par exemple, l'étude de la maladie de Huntington a d'abord montré le caractère familial de celle-ci. Ensuite, une analyse des arbres généalogiques a montré qu'elle était liée à un gène dominant. Puis, ce gène a été identifié. L'étape suivante serait logiquement d'étudier les fonctions de ce gène.

2) Le conflit. Parfois, deux (ou plus) traditions intellectuelles et méthodologiques arrivent à des résultats différents pour une même question de recherche. Toute nouvelle étude de cette question doit témoigner du conflit et y prendre position, à moins d'arriver à réconcilier les parties. Par exemple, l'exposition prénatale au tabagisme estelle un facteur de risque pour le développement de l'hyperactivité/inattention, ou un même risque génétique prédispose-t-il à la fois au tabagisme et à l'hyperactivité/inattention? Des résultats vont dans les deux sens. Si vous étudiez cette question, vous aurez à choisir un camp sur la base de vos résultats.

3) L'obstacle à franchir. Parfois, la recherche dans un domaine se heurte à un obstacle méthodologique: une explication nous échappe, ou encore une manière de mesurer ou de modéliser le phénomène d'intérêt. Pensez à la découverte de la structure de l'ADN par l'équipe de Watson et Crick, à tout ce qui est devenu possible ensuite. À un niveau 
plus modeste, la création ou l'adaptation d'un outil de mesure de traits ou de comportements pourrait s'inscrire dans une intrigue d'obstacle.

Figure 1. Modèle d'intrigue.

La quête

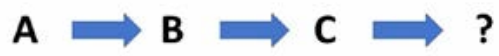

\section{À vous d'amener $D$}

Le conflit

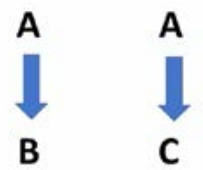

À vous de prendre position ou de résoudre le conflit

L'obstacle

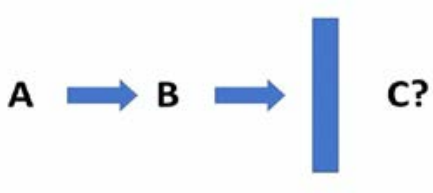

À vous d'abattre l'obstacle

II y a d'autres intrigues possibles et je vous inviter à consulter Silvia (2015) en complément (Silvia les appelle des gabarits rhétoriques, à retenir si vous trouvez qu'«intrigue» ne fait pas assez savant). Essayez de placer vos idées dans la logique d'une de ces intrigues. Une cohérence devrait commencer à apparaitre en jouant avec les possibilités et vous allez la transformer en structure. À la lumière des intrigues possibles, réfléchissez à ce que nous savons de votre sujet de recherche et à ce que nous ne savons pas. Est-ce qu'il nous manque la prochaine pièce du casse-tête? Un argument pour trancher entre deux positions? Une manière de modéliser le phénomène? La réponse à ces questions ne doit pas être prise à la légère. Elle déterminera la structure de votre introduction, la formulation finale des objectifs de recherche et la manière de rapporter l'interprétation des résultats. Elle vous permettra également de sélectionner les références qui soutiennent l'histoire que vous allez raconter et à mettre de côté les autres sans faire une crise d'anxiété.
Un dernier point: il y a certainement plusieurs intrigues qui rendent compte du contexte dans lequel vous allez développer vos questions de recherche. Où je vois un conflit, peut-être voyez-vous plutôt de la continuité. Le choix que vous ferez fait partie de l'originalité de votre contribution, mais détermine aussi la rigueur de votre recension des écrits.

\section{Structurer l'écrit}

Après l'effervescence de la recherche des idées et de leurs liens, il est temps de passer à la pensée convergente, de choisir une intrigue comme base de structure et de la développer. II reste à donner à I'histoire une forme linéaire, donc à situer le début, le milieu et la fin pour finaliser un plan d'écriture (vous n'alliez pas écrire sans plan, n'est-ce pas?). J'emprunte ici le modèle de Schimel (2012), auteur de I'incontournable Writing Science. L'article scientifique classique s'articule naturellement sur ce qu'il appelle le modèle OCAR, pour Ouverture, Challenge, Action et Résolution. L'ouverture établit l'importance du sujet de recherche, le pourquoi; le "challenge», ou défi, pose la question de recherche dans le contexte de l'ouverture; l'action décrit ce qu'on a fait pour relever le défi; la résolution interprète les retombées de I'action dans le contexte de l'ouverture.

Vous voyez immédiatement comment la structure OCAR correspond bien au moule IntroductionMéthode-Résultats-Discussion, mais il y a plus. Chacune de ces sections, et chacune de leurs sous-sections, doivent présenter clairement un arc narratif, soit le trio ouverture (pourquoi est-ce important de dire ce que vous dites), action (le dire) et résolution (dire à vos lecteurs et lectrices quoi en retenir pour bâtir votre argumentation). L'arc narratif est donc très similaire à la structure OCAR, mais sans le challenge. Schimel fait un parallèle entre la structure d'un écrit scientifique et une série télé: Vous avez une histoire qui se poursuit à travers tous les épisodes, et des arcs secondaires qui peuvent se résoudre en un ou deux épisodes. Pensez à une série policière relatant l'enquête sur un meurtre jusqu'à l'arrestation du coupable, avec des arcs secondaires comme un malentendu entre deux enquêteurs ou la liaison illicite entre un chef de police et un procureur. Chaque arc narratif débute à moment précis et connaît un développement suivi d'une résolution (si la série est bien écrite!). 
J'insiste ici pour dire que l'arc narratif n'est pas une notion aussi abstraite qu'il n'y apparait. Les éléments de l'arc narratif sont mes principaux outils d'analyse de la structure d'un écrit scientifique (voir Tableau 1). Quand le fil narratif s'effiloche, c'est généralement parce qu'il manque au moins un des éléments d'un arc. En terminant votre plan, assurez-vous qu'il contient un grand arc (la structure OCAR) et une succession d'arcs complets. Quand votre superviseur(e) relit votre texte et note qu'il "manque quelque chose», ce qui manque n'est pas forcément une information, mais un élément de structure sans lequel le sens du passage est obscur; pourquoi avez-vous amené une idée (ouverture)? Ou comment contribue-t-elle à votre argumentation (résolution)? Sans arcs, votre article ressemblera à une liste d'épicerie: une succession d'idées dont les liens s'avèrent incompréhensibles. À noter que c'est à vous de rendre les liens explicites, pas aux lecteurs et lectrices de les deviner.

Ce n'est pas encore concret? Décortiquons la structure OCAR et les arcs narratifs que chaque section de l'écrit scientifique comprend.

\section{Tableau 1}

Arcs narratifs dans les différentes sections de l'écrit scientifique

\begin{tabular}{lllll}
\hline Arc & Introduction & Méthode & Résultats & Discussion \\
\hline Ouverture & $\begin{array}{l}\text { Importance du } \\
\text { problème }\end{array}$ & $\begin{array}{l}\text { Contexte: } \\
\text { échantillon }\end{array}$ & $\begin{array}{l}\text { Rappel de chaque } \\
\text { question }\end{array}$ & $\begin{array}{l}\text { Rappel du } \\
\text { problème }\end{array}$ \\
Action & Ce que l'on sait & $\begin{array}{l}\text { Procédure et } \\
\text { instruments }\end{array}$ & $\begin{array}{l}\text { Résultats des } \\
\text { analyses }\end{array}$ & $\begin{array}{l}\text { Interprétation: } \\
\text { réponse aux } \\
\text { questions }\end{array}$ \\
Résolution & $\begin{array}{l}\text { Ce qui manque: } \\
\text { challenge }\end{array}$ & Choix des analyses & Inférences directes & $\begin{array}{l}\text { Changements au } \\
\text { contexte théorique }\end{array}$ \\
\hline
\end{tabular}

\section{Structurer l'introduction}

Si vous n'avez reçu qu'un seul conseil concernant la structure de votre introduction, c'est de la construire en entonnoir: du général vers le particulier. Fort bien, mais trop vague pour être utile! Une autre approche s'appelle CARS pour Create A Research Space: montrer ce qui manque dans la recherche de votre domaine pour justifier la pertinence de votre travail (Sword, 2012). C'est déjà plus précis, mais encore insuffisant si le manque décelé s'avère superficiel. Par exemple, n'allez pas dire que la question de recherche n'a été explorée qu'avec des enfants de 10 ans et que vous innovez parce que vos participants en ont 12 ! Pour créer une structure d'introduction qui donnera à vos lecteurs ou lectrices l'envie de continuer à lire, vous devrez intégrer votre intrigue dans un arc narratif.

La structure d'introduction proposée au Tableau 1 ressemble à l'approche CARS; vous allez montrer ce qui manque, mais à l'aide de la réflexion qui a mené au choix de votre intrigue. L'ouverture doit situer la problématique plus large: celle qui intéresserait votre grand-mère si vous lui décriviez votre recherche, la question importante qui a des répercussions dans la vie des êtres humains, celle qui commence par pourquoi et non par comment. Prenez un moment de recul pour vous rappeler de cette question et peut-être pour rallumer votre curiosité initiale.

L'action de l'introduction, c'est l'articulation de ce qu'on sait du problème sous forme d'intrigue. Donc, si vous jugez que votre sujet correspond au modèle de la quête, vous devrez présenter la succession de résultats des recherches précédentes en mettant en valeur leur continuité. Si vous avez vu un conflit, vous devrez articuler clairement les positions opposées en mettant l'accent sur les contradictions dans la recherche passée et sur leurs causes. Si vous suivez l'intrigue de l'obstacle, décrivez en quoi celui-ci bloque la continuation de la recherche et ce qui manque pour le contourner. L'intrigue vous amène à déterminer un manque qui n'a rien de superficiel: quelle est la prochaine étape dans la continuité, I'information qui permettrait de résoudre le conflit ou la méthode qui doit être développée pour contourner l'obstacle? L'énoncé explicite de ce manque constitue la résolution de l'introduction. 


\section{Le Challenge}

Après avoir accompli les étapes précédentes de structuration de l'introduction, vous devriez comprendre votre projet avec une profondeur nouvelle. Vous pouvez donc maintenant déterminer la formulation finale de vos questions de recherche spécifiques. Elles devraient apparaître aux lecteurs et lectrices comme la conséquence logique de la résolution de l'introduction. Les questions de recherche font partie de l'introduction dans la plupart des écrits scientifiques, mais occupent leur propre place dans la structure OCAR.

\section{La méthode}

La section méthode est rigidement codifiée. Alors, la meilleure stratégie consiste à suivre le modèle offert par les articles les plus pertinents de votre liste de références. Notez toutefois que la structure habituelle suit un arc narratif (voir Tableau 1). Elle commence par le contexte: sur qui porte la recherche, donc les participants (humains ou autre). Ensuite, pour l'action, qu'avez-vous fait avec les participants, et avec l'aide de quels instruments avez-vous récolté les données? En résolution, qu'allez-vous faire avec ces données pour répondre à vos questions?

La section de description des analyses, s'il y en a une, arrive donc logiquement à la fin. Ne négligez pas cette sous-section si vous vous apprêtez à entraîner vos lecteurs et lectrices dans une jungle touffue d'analyses et de résultats: elle leur donnera la carte pour s'y retrouver. Mieux encore, elle vous donne le plan pour construire la section des résultats.

\section{Les résultats}

Si un deuxième conseil de rédaction vous a été donné, c'est celui de ne rien interpréter dans la section résultats lorsque vous présentez des analyses quantitatives (certains articles scientifiques combinent les résultats et la discussion, mais ils sont rares en psychologie). Ce conseil est juste, mais souvent mal interprété: il ne s'agit pas de présenter une avalanche de chiffres en espérantqu'ils parlerontd'eux-mêmes. Pourcompléter ce conseil, il faut distinguer les inférences des interprétations (Schimel, 2012). Les inférences sont les conséquences directes de l'analyse que vous rapportez, que toute personne familière avec l'analyse serait en mesure d'énoncer sans même connaître la question de recherche. Par exemple, si vous faites un test $t$ et qu'il n'est pas significatif, vous pouvez inférer qu'il n'y a pas de différence de moyennes entre deux groupes. L'interprétation de cette inférence fait appel au contexte de la problématique et à l'intrigue. Si les interprétations appartiennent à la discussion et à la conclusion, les inférences appartiennent à la section des résultats.

L'arc narratif sauvera vos lecteurs et lectrices de I'avalanche de chiffres. Idéalement, la section résultats contient plusieurs sous-sections. Après un survol des analyses descriptives, qui confirment que vous avez le nombre et la variance nécessaires pour continuer, je suggère de créer une sous-section pour chaque question de recherche. Certains utilisent même les principaux éléments de chaque question comme sous-titres (voir p. ex., Agnew-Blais, Polanczyk, Danese, Wertz, Moffitt, \& Arseneault, 2016), ce qui donne l'ouverture d'entrée de jeu en rappelant les raisons pour lesquelles vous avez fait cette analyse. Ensuite vient l'action: quelle analyse a été réalisée et quelles données statistiques a-t-elle livrées. C'est le moment de montrer vos $r, F$, p, etc. et de diriger les lecteurs et lectrices vers les tableaux et figures. Enfin, en résolution, donnez l'inférence: I'association était significative, il y avait une différence de moyennes entre tel et tel groupe, mais pas tel autre, la structure factorielle était confirmée... Ce que vos lecteurs ou lectrices ont besoin de retenir pour passer à l'interprétation.

Dernier détail: assurez-vous de la cohérence exacte de la section résultats avec la description des analyses de la section méthode, tant au niveau des analyses rapportées que de leur ordre.

\section{La discussion}

Quand un.e étudiant.e venait me voir avec de la difficulté à écrire sa discussion, je commençais par demander à lire son introduction. C'est le secret de la discussion: elle représente l'image miroir de I'introduction, un entonnoir inversé qui va du particulier vers le général. Assurez-vous donc, avant de procéder, que l'arc narratif de l'introduction est bien structuré autour de l'intrigue. Autrement, vous risquez de commettre l'erreur classique de réécrire les résultats avec davantage de mots. 
Une bonne discussion représente un travail ardu, mais grandement facilité par une bonne planification de la structure complète de l'écrit. Commencez par rappeler brièvement le problème et les questions soulevées en ouverture. L'essentiel de l'action consiste à répondre aux questions en interprétant les inférences. Par exemple, si vous posiez une question sur les différences potentielles entre plusieurs groupes pour une mesure donnée, avez-vous trouvé cette différence? Assurezvous $d$ 'interpréter toutes les inférences pour répondre à toutes les questions et sous-questions, mais sans répéter inutilement les statistiques ou autres inférences. C'est la première partie de l'interprétation, mais ne vous arrêtez pas en si bon chemin; vous devez maintenant interpréter pour vos lecteurs et lectrices la signification des réponses à toutes les questions mises ensemble. Pour cette étape, la plus passionnante de l'écrit scientifique, car elle donne la mesure de votre contribution, reprenez votre intrigue. Avec l'ensemble de votre travail, avez-vous ajouté un jalon à la chaîne des découvertes? Pris position dans le conflit? Aplani un obstacle? Exécuté toute autre action appelée par une autre intrigue? Sinon, que s'est-il passé? Répondez à ces questions pour vousmême... et ensuite pour vos lecteurs et lectrices. J'espère que vous y trouverez une satisfaction intellectuelle aussi grande que les efforts déployés pour atteindre cette étape.

Ce n'est pas encore terminé... vous arrivez à la résolution de la discussion, qui bouclera la boucle commencée en début d'introduction. Vous avez situé votre recension des écrits et vos questions dans la problématique plus large qui lui donne son importance. Les résultats qui vous ont permis de donner une réponse aux questions, même non concluante, modifie cette problématique pour les autres chercheurs et chercheuses de votre domaine. Montrez - modestement - de quelle façon le contexte théorique a changé à la suite de votre étude. Si vous avez des questions de recherches spécifiques, originales et découlant directement de votre étude à proposer, allez-y. Si les seules propositions que vous avez à faire à vos collègues sont de l'ordre de "refaire la même étude avec un plus gros $N$ », mieux vaut vous abstenir... ou poursuivre votre réflexion.

\section{La conclusion}

Pour Schimel (2012), la conclusion est la véritable résolution de l'écrit scientifique. Votre mémoire ou thèse en comprendra une, mais tous les éditeurs de revue scientifique n'en exigent pas pour les articles. Que votre conclusion soit une section identifiée comme telle ou un simple paragraphe à la fin de la discussion, elle est cruciale pour modeler ce que les lecteurs et lectrices retiendront de votre écrit. Schimel suggère de parcourir en conclusion toute la boucle, du contexte théorique à l'interprétation des résultats dans leur ensemble, puis retourner au contexte et le dépasser en montrant le nouveau point de départ suggéré par votre étude. Soigner la conclusion va vous permettre de convaincre les lecteurs qui commencent à lire par la fin (vous l'avez déjà fait, non?) de porter attention à votre étude.

\section{Le résumé}

Il s'agit du premier contact des lecteurs et lectrices avec votre écrit scientifique, et de la dernière section que vous devriez rédiger. Le résumé représente une mini-histoire dans laquelle se retrouvent tous les éléments de la structure, soit l'ouverture, le défi, l'action et la résolution, parfois en respectant une limite de mots cruelle. Certains auteurs, dont Schimel (2012), s'étendent sur l'importance de la première phrase de tout écrit pour "ferrer le poisson» et donner envie de continuer à lire... mais dans un écrit scientifique, c'est vraiment le résumé qui joue ce rôle. Attendez de posséder à fond votre recherche, son contexte et ses implications avant d'écrire le résumé pour éviter des souffrances inutiles, à vous autant qu'à vos lecteurs.

Maintenant, peaufinez votre bibliographie et préparezvous... à réviser. Mais avant d'aborder ce sujet crucial entre tous, j'aimerais vous inviter à faire un exercice pour concrétiser ce que j'ai tenté de vous expliquer sur la structure. Prenez un article scientifique que vous trouvez clair et agréable à lire. Cherchez dans cet article les éléments de la structure OCAR, identifiez les arcs narratifs à l'intérieur des sections. Examinez de quelle manière les idées sont structurées et les paragraphes coulent les uns dans les autres. Schimel (2012) note que les scientifiques qui influencent durablement leur domaine ont des talents en recherche ET en communication. Donc, rendez-vous service en prenant leurs écrits comme modèles. Rappelez-vous: le temps investi dans la préparation de l'écriture rapporte en temps gagné lors de la rédaction et en satisfaction. 


\section{Réviser l'écrit}

Qu'il s'agisse d'une thèse, d'un mémoire ou d'un article, vous écrivez pour être lu(e) par des scientifiques professionnels. Une qualité de rédaction tout aussi professionnelle est donc attendue. Malheureusement, la plupart d'entre vous arrivent aux études supérieures sans avoir reçu une formation adéquate en la matière. De plus, dans la plupart des programmes, il n'y a pas de cours de rédaction et vous êtes laissé(e) à vousmême pour vous rattraper, avec le soutien d'un(e) superviseur(e) plus expérimenté(e), mais pas plus formé(e) que vous. Voici donc le secret que vous devez connaître: la différence entre un texte amateur et maladroit et un texte professionnel tient dans la révision. Fini, le temps où vous pouviez envoyer un travail de session à la dernière seconde sans l'avoir même relu! Je devrais parler des révisions, parce qu'il y en aura beaucoup et à plusieurs niveaux. En fait, vous passerez plus de temps à réviser et à réécrire qu'à écrire, donc planifiez votre temps en conséquence! J'y reviendrai.

\section{Réviser seul(e)}

Vous êtes, bien sûr, la personne responsable au premier chef de la révision de votre écrit. Une première révision avant de faire lire votre article à qui que ce soit (de grâce, n'infligez jamais à personne la première version sans révision!) concerne la qualité scientifique: votre introduction représente-elle bien les écrits scientifiques du domaine? Avez-vous rapporté fidèlement vos résultats, même ceux qui vous déçoivent? Votre interprétation est-elle juste dans le cadre théorique proposé? Voilà un bon moment pour mettre à jour votre recension des écrits. Si votre structure est basée sur une intrigue adéquate, vous arriverez à y inclure toute nouvelle étude pertinente parue pendant la rédaction.

La révision de la structure fine du texte et de l'usage de la langue sont aussi importants que la révision scientifique pour communiquer vos idées. Ne cherchez pas de raccourci: il vous faut réviser ligne par ligne et phrase par phrase en vérifiant la grammaire, la syntaxe, le choix du vocabulaire, la conjugaison et la ponctuation. Je vous rappelle ici que les professionnels de la rédaction ont constamment le nez dans leurs livres de référence. Vous n'êtes pas dispensé(e) de consulter grammaires et dictionnaires.
Un autre aspect de la révision fine est la chasse au verbiage. Un de mes professeurs de maîtrise suggérait de couper un mot par phrase. C'est presque toujours possible (j'ai coupé trois mots dans cette phrase)! On s'aperçoit vite que des phrases entières du premier brouillon s'avèrent superflues. En fait, si vous n'avez pas modifié chaque phrase, vous n'avez probablement pas assez révisé.

Quand réviser? Mieux vaut prévoir du temps, quelques jours au moins, pour vous changer les idées et oublier ce que vous avez écrit. Autrement, vous lirez des mots absents ou serez aveugles à des mots oubliés dans une phrase après une modification de la structure. Bref, certains auteurs (Wilbers, 2000) suggèrent de désigner un tiroir de bureau (pour notre époque, un répertoire sur un disque dur) comme «congélateur» où entreposer les articles quelques temps avant de réviser.

\section{Réviser avec d'autres}

Bien entendu, la révision par votre superviseur(e) aura une grande influence sur le produit final: il ou elle a participé à la conception du projet de recherche et reste votre principal(e) conseiller ou conseillère scientifique. Toutefois, cette force représente également la principale faiblesse du processus de révision: votre superviseur(e) connaît tellement votre projet qu'il ou elle risque de faire des liens entre les idées alors qu'ils ne sont pas explicitement présents dans le texte. Pour la révision de la structure, une bonne solution consiste à réviser aussi avec vos pairs. La personne idéale dispose de la formation nécessaire pour comprendre votre projet, sans être familière avec celui-ci. Elle se montrerait critique autant que bienveillante: elle devrait faire preuve de tact, mais sans craindre de signaler les endroits dans la structure du texte où elle perd le fil de votre raisonnement, les expressions maladroites, les phrases trop longues. J'ai proposé sur mon blogue (Forget-Dubois, 2017; voir Annexe A) une série de questions auxquelles pourrait répondre votre réviseur(e) volontaire. Vous lui rendrez la pareille. $\mathrm{Si}$ on prenait la rédaction au sérieux, il y aurait des clubs de révision dans toutes les facultés.

Suivront alors des révisions avec votre comité de suivi, votre comité d'évaluation, les réviseurs scientifiques si vous écrivez un article... courage! Par ailleurs, il y a un défi à chercher la bonne manière d'exprimer une 
idée que vous trouverez peut-être stimulante et je ne voudrais pas vous donner l'impression que la révision représente une période toute noire. Comme pour le reste, la prévoir, la planifier et la réaliser méthodiquement vont rendre la révision plus courte et plus agréable.

\section{Gérer ses comportements d'écriture}

Écrire un mémoire, une thèse ou un article scientifique est long et difficile, même quand on a la chance de prendre plaisir à l'exercice. Pour la plupart des étudiants de maîtrise ou de doctorat, il s'agit du plus long texte suivi jamais rédigé, sans formation préalable. Avant de vous lancer dans la rédaction, prenez le temps d'attacher votre parachute, c'est-à-dire de planifier: vous aurez besoin d'un plan du texte, d'un plan pour le rédiger et d'un plan pour rester discipliné et motivé.

\section{Planifier l'écrit}

J'ai fait allusion à la nécessité de faire un plan incluant les arcs narratifs de votre thèse, mémoire ou article. Ne sautez pas cette étape: vous avez un texte professionnel à rendre, et non un travail de session. Jeter les idées sur papier à mesure qu'elles viennent ne suffira pas. Un bon plan prend du temps à développer, mais c'est du temps économisé de nombreuses fois lors de la rédaction. En situant les éléments du texte sous forme d'arcs narratifs de prime abord, vous allez vous assurer de la suite logique du fil narratif de l'ensemble. Le plan, c'est choisir une intrigue et s'en servir pour imposer une structure linéaire à la complexité qui a émergée de vos lectures et de votre jeu avec les idées. N'hésitez pas à écrire "ouverture», «action » et «résolution» dans le plan aussi souvent que nécessaire, sans oublier de rendre l'intrigue explicite. Dans le texte, vous n'écrirez évidemment pas que vous suivez le modèle de "la quête», mais dans le plan, c'est possible. Vous pouvez même prévoir les liens entre les paragraphes et la structure des paragraphes (voyez Schimel (2012) pour les techniques de structure des paragraphes et d'enchaînement). N'oubliez pas $d^{\prime}$ intégrer également les références et de situer les tableaux et figures.

II faut que je précise ici que j'aime travailler à l'élaboration d'un plan. À cette étape, le jeu intellectuel avec les idées prime et la créativité s'exprime. Toute cette énergie dépensée à lire des articles scientifiques et à bûcher sur des analyses se voit enfin justifiée. J'aime tellement planifier que toute ma technique d'écriture est basée sur le plan: écrire un plan global d'abord, puis y ajouter des détails, repasser pour ajouter d'autres détails... jusqu'à ce que je puisse écrire le plan en phrases, ce que je fais en attaquant la rédaction d'une section de texte pour avoir rapidement un squelette de texte cohérent. Ensuite, je repasse sur le texte pour mettre de la chair sur le squelette. Puis je repasse encore et encore... j'aime aussi réviser, pendant un temps du moins, donc je révise beaucoup et souvent. Comme je vous le disais plus tôt, il faut trouver une méthode de rédaction qui convienne à sa personnalité et ceci fait mon bonheur.

Prendre le temps de perfectionner un plan détaillé représente un énorme avantage: si vous êtes capable de compléter un plan, vous serez capable d'arriver au bout de la thèse, du mémoire ou de l'article. Par ailleurs, si vous n'arrivez pas à faire ce plan, vous n'êtes pas prêt(e) à écrire... et vous lancer dans la rédaction quand même risquerait de vous conduire au découragement, voire à l'échec. Un bon plan tue aussi le syndrome de la page blanche, parce que vous savez toujours ce que vous avez à rédiger. Je ne suis pas en train de vous dire que la rédaction assistée d'un plan sera facile, mais facilitée. Par ailleurs, vous n'êtes pas prisonnier de votre plan en cours de rédaction. Toutefois, assurezvous qu'un changement va s'inscrire dans un arc narratif et dans la structure OCAR.

\section{Planifier la rédaction}

Vous connaissez sans doute le précieux «assieds-toi et écris ta thèse " de Geneviève Belleville (2014; sinon, informez-vous). Comme elle l'explique sagement, il y a une règle d'or pour arriver à rédiger: bloquer des plages de temps consacrées à la rédaction et les respecter scrupuleusement. Cette règle de base se complète de plusieurs autres: placer les plages de rédaction aux moments de la journée où vous êtes le plus productif; prévoir des plages ni trop longues ni trop courtes, en lien avec votre confort; faire taire les sources de distraction; ne pas oublier de prendre des vacances de rédaction à l'occasion.

Que faire durant les plages de rédaction? Atteindre des objectifs. Respecter la plage de temps peut-être 
un objectif en soi, mais vous avez besoin d'objectifs à court et à long terme. Puisque vous avez fait un plan détaillé, vous pouvez le découper en jalons à placer dans vos plages de temps. Ainsi, l'objectif d'une période de rédaction sera précis et atteignable: non pas «écrire l'introduction», mais «écrire l'ouverture et l'action de la deuxième sous-section de I'introduction ». Terminer l'introduction est un objectif à plus long terme. Déterminer des objectifs réalistes demandera des ajustements en cours de route, à mesure que vous vous connaîtrez mieux comme rédacteur ou rédactrice. Par ailleurs, n'oubliez pas de prévoir votre révision dans votre planification du temps de rédaction. Vous ne contrôlez malheureusement que votre propre temps de révision. Cette dernière peut s'étendre sur plusieurs mois de plus quand votre texte se trouve entre les mains des membres de votre comité et des réviseurs scientifiques d'une revue: profitez de l'expérience de vos pairs et de vos superviseur(e)s pour estimer le temps à prévoir.

Planifier les moments de rédaction ne peut que vous aider à voir un jour la fin de votre programme d'études, mais à quel point cette planification doit être minutieuse dépend de vous et des circonstances de votre vie. Si rédiger est votre principale activité pendant quelques mois et que vous aimez écrire, la planification serrée des plages de temps est moins importante que si vous jonglez avec la rédaction, un travail à temps partiel et des triplés âgés de 18 mois.

Un dernier mot sur la planification de votre rédaction: portez attention à l'ergonomie de votre environnement de travail. Assurez-vous que la hauteur de votre table est adéquate, procurez-vous une bonne chaise, ajoutez un écran, un clavier et une souris à votre ordinateur portable. La pose «bossu de Notre-Dame» au-dessus de celui-ci pendant des mois vous vaudra un abonnement à vie en physiothérapie, croyez-moi sur parole.

\section{Quelques aménagements}

Je le répète, écrire est un acte très personnel et votre objectif devrait être la construction de votre propre méthode. Par exemple, que signifie pour vous respecter une plage de rédaction? Garder les fesses sur la chaise et les doigts sur le clavier? C'est un bel idéal, mais cela ne l'est pas si vous butez sur un passage difficile et sentez poindre des larmes de frustration. Mieux vaut, à mon avis, faire quelques pas dans la pièce, arroser les plantes ou exécuter toute autre activité brève ne requérant pas une grande énergie intellectuelle ou émotive. Après cette petite pausesanté pour votre cerveau, vous pourrez poser un œil neuf sur le problème. Je ne vois pas ces activités comme une entorse au respect d'une plage de temps, du moins si les objectifs de rédaction sont ordinairement atteints. Il semble d'ailleurs que le « réseau du mode par défaut» du cerveau continue à fonctionner pendant la pause, c'est pour cette raison que la solution à notre problème semble parfois surgir quand on reprend le travail (Kaufman \& Grégoire, 2015). Quoiqu'il en soit, respecter la plage de rédaction ne devrait pas représenter une source de stress, mais une habitude de travail saine. Si vous ressentez toutefois le besoin d'une structure moins souple, les techniques du genre pomodoro ${ }^{13}$ sont pour vous. Éviter d'en venir à détester sa rédaction aide à la terminer.

De même, la manière de rédiger varie d'une personne à l'autre. Certains auteurs vous encourageront à «bulldozer» à travers la page plutôt qu'à "poser des briques» (Wilbers, 2000; Belleville, 2014), c'est-à-dire avancer la rédaction sans égard au style et aux fautes pour faire grimper rapidement le nombre de pages. La révision vient plus tard. Si cette méthode vous convient, tant mieux... Personnellement, bulldozer me rend malheureuse. Je préfère écrire un paragraphe à la fois et ne passer au suivant que lorsque je suis assez satisfaite du premier, sachant que je le réviserai encore. D'ailleurs, la méthode est toujours la même: alterner une phase de rédaction et une phase de révision. Tout ce qui change, c'est la durée de la rédaction avant de passer à la révision. Encore une fois, la méthode qui vous permet d'atteindre vos objectifs de rédaction tout en préservant votre bonne humeur est la bonne: si vous avez essayé sans succès de bulldozer, pourquoi ne pas poser des briques? Écrire s'apprend, notamment en essayant les méthodes des autres, mais sans subir un stress supplémentaire en essayant de s'y conformer.

D'ailleurs, inclure des éléments positifs dans votre méthode d'écriture est crucial pour l'aboutissement du projet. II ne s'agit pas de dévorer de la crème 
glacée à chaque objectif de rédaction atteint: cocher un item dans une liste de tâches peut vous donner une petite satisfaction quotidienne. Lors de la rédaction de ma thèse, je me fixais un objectif hebdomadaire. Si je n'arrivais pas à l'atteindre, je devais travailler le samedi; si je l'atteignais d'avance, j'avais congé le vendredi après-midi. Je n'ai pas pris beaucoup de congé, mais je n'ai pas travaillé souvent le samedi non plus. Voyez donc ce qui vous motive. Bien sûr, une grosse récompense en fin de rédaction peut sembler alléchante, mais restez réaliste. Allez-vous vraiment annuler un voyage en Asie si vous ne terminez pas à la date fixée?

Finalement, pour votre bien-être et pour la qualité du résultat, essayez de garder une attitude positive envers votre écrit scientifique. Pensez à un article scientifique que vous avez trouvé stimulant et bien écrit. Peut-il avoir été rédigé dans un état d'esprit du genre «je déteste écrire, je veux être ailleurs, j'aurais dû devenir instructeur de yoga à la place»? Les écrits scientifiques ne sont pas dénués d'émotion. Votre texte peut communiquer votre enthousiasme pour votre sujet de recherche... ou l'apathie ressentie dans le processus de rédaction.

\section{Du bon usage des conseils de rédaction}

On peut toujours apprendre à améliorer son écriture. Pour ma part, je voudrais avoir un jour la plume assez légère pour écrire à nouveau cet article avec moins de verbes conjugués à l'impératif. Je n'ai que des conseils et des suggestions: vous pouvez très bien terminer votre thèse avec succès sans eux. Toutefois, pour ceux et celles que la rédaction écrase, pourquoi ne pas essayer de les mettre en pratique? Et si mes méthodes ne vous conviennent pas, vous aurez appris à mieux vous connaître comme rédacteur ou rédactrice et pourrez réinvestir ces connaissances dans l'élaboration d'une méthode qui vous réussira mieux. Pigez dans les références ci-dessous, et dans leurs listes de références. Peu importe la méthode, du moment que vous en avez une et qu'elle soutient vos objectifs en vous gardant raisonnablement heureux ou heureuse. Tout au long du processus, observezvous sans complaisance, mais avec bienveillance, pour réduire l'incidence de vos faiblesses et capitaliser sur vos forces. Surtout, et je me permets un dernier impératif, construisez votre méthode autour des aspects de la recherche et de la rédaction que vous aimez. Une thèse écrite avec plaisir en communiquera aux lecteurs et lectrices et vous laissera un bien meilleur souvenir.

\section{Références}

Agnew-Blais, J. C., Polanczyk, G. V., Danese, A., Wertz, J., Moffitt, T. E., \& Arseneault, L. (2016). Evaluation of the persistence, remission, and emergence of attention-deficit/hyperactivity disorder in young adulthood. JAMA Psychiatry, 73(7), 713-720. https:// doi.org/10.1001/jamapsychiatry.2016.0465

Belleville, G. (2014). Assieds-toi et écris ta thèse! Trucs pratiques et motivationnels pour la rédaction scientifique. Québec: Presses de l'université Laval.

Forget-Dubois, N. (2017). N'essayez pas ça (seul) à la maison: la révision d'un article scientifique. https:// laplumescientifique.com/2017/10/09/nessayez-pasca-seul-a-la-maison-la-revision-dun-article-scientifique/

Kaufman, S. B. \& Gregoire, C. (2015). Wired to Create: Unraveling the Mysteries of the Creative Mind. New York, NY: Perigee.

Schimel, J. (2012). Writing Science: How to Write Papers That Get Cited and Proposals That Get Funded. New York, NY: Oxford.

Silvia, P. J. (2015). Write it up! Practical Strategies for Writing and Publishing Journal Articles. Washington, DC: American Psychological Association.

Sword, H. (2012). Stylish Academic Writing. Cambridge, MA: Harvard University Press.

Wilbers, S. (2000). Keys to Great Writing: Mastering the Elements of Composition and Revision. Cincinnati, $\mathrm{OH}$ : Writer's Digest Books.

\section{Annexe A}

Quelques questions à poser à un pair qui révise votre écrit scientifique:

- Mes questions de recherche sont-elles explicitement formulées?

- Mes questions de recherche apparaissent-elles comme l'aboutissement logique de l'introduction? Ai-je démontré ce qui manquait dans la littérature que ma recherche contribue à combler? 
- Ai-je confondu la fin et les moyens en formulant mes questions de recherche en fonction de la méthode et non en fonction du contexte théorique?

- Pourrais-tu refaire les étapes de ma collecte de données/expérience/etc. ?

- Comprends-tu facilement le lien entre chaque résultat rapporté et la question de recherche (et ses hypothèses)?
- Pourrais-tu décrire l'apport à l'article de chaque tableau et figure?

- Est-ce que je répète les résultats dans la discussion? Est-ce que je me répète souvent, dans tout le texte?

- Ai-je répondu aux questions que j'ai soulevées dans l'introduction?

\section{Pour citer l'article}

Forget-Dubois, N. (2020). L'art de ne pas réinventer la roue: mener à bien un projet de rédaction scientifique. Psycause: Revue scientifique étudiante de l'École de psychologie de l'Université Laval, 10(1), 61-71.

\section{Droits d'auteur}

(C) 2020 Forget-Dubois. Cet article est distribué en libre accès selon les termes d'une licence Creative Commons Attribution 4.0 International (de type CC-BY 4.0) qui permet l'utilisation du contenu des articles publiés de façon libre, tant que chaque auteur ou autrice du document original à la publication de l'article soit cité(e) et référencé(e) de façon appropriée. 